\section{The Compton Effect.}

Apropos of the very readable review of the discussion on scattered X-rays at the Toronto meeting of the British Association, published in Nature of October 25 , p. 627 , we presume your readers may be interested in a bit of new evidence on the reality of the Compton effect, obtained since that meeting.

We have photographed spectra of scattered molybdenum rays, with the tube and scatterer in one room and the spectrograph in another. The walls near the tube and scatterer were lined with lead, and the room was large enough to remove all other substances exposed to the rays to a safe distance. By that we mean a distance such that even if those substances converted all the radiant energy falling on them into tertiary rays, the total quantity of tertiary radiation sent by them to the "scatterer" would be reduced by the inverse square law alone far beyond detection. We built two such sets of apparatus, in different parts of the room, and used them at different times. One was a molybdenum-target tube with a sulphur scatterer. The other was another molybdenumtarget tube, of a different form, with an aluminum scatterer, using a spectrograph of quite a different design.

Both of these sets of apparatus gave spectra like those described previously by Ross (Phys. Rev., Nov. I923; Proc. Nat. Acad. Sci., July I924), which had given the first definite proof of the existence of the Compton effect in elements other than carbon. These new spectra showed the Compton-theory lines as strong as in the earlier spectra. Apparently, therefore, these experiments confirm the calculations described at the Toronto meeting showing that those spectra were quite free from any contamination by box effects, and were due only to the elements to which Ross ascribed them. D. L. WEBster.

Stanford University, California, U.S.A.,

$$
\text { P. A. Ross. }
$$

December 3 .

\section{Arsenic in Oysters.}

Your contributor J. S. G. in reviewing (NATURE, December 20, p. 9I3) the report of the Ministry of Agriculture and Fisheries on oyster mortality in I920, and dealing with the finding of 3.7 parts of arsenic per million in oysters from certain beds, comments on the serious questions raised by such a fact. A definite pronouncement is needed as to what constitutes danger. As is well known, the Royal Commission on Arsenical Poisoning, in its report in 1903 , recommended that no substance used in the preparation of food should contain more than I/roo grain per lb. This recommendation has been adopted as a standard for years, and many prosecutions have been successful for quantities but little in excess of this. Surely the position is now more illogical than ever it was. Is the fishmonger to be prosecuted if his oysters have three parts per million ( $1 / 50$ grain per lb.), and, if not, why should a grocer be charged if, say, a baking powder-probably not made by himself-contains this amount?

Results of investigation in Sweden show that fish may have an arsenic content up to four parts per million, and my own analyses of fish sold in the London market, recently communicated to the Society of Public Analysts, confirm this figure in respect of certain plaice. The administration of the Sale of Food and Drugs Acts in this respect requires revision.

The Laboratory, i I Billiter Square, H. E. Cox.

London, E.C.3, December 24

NO. 2880 , vOL. I I 5 ]

\section{Convective Equilibrium.}

THERE is a rather important consideration connected with the production of cloud in rising air currents (which does not seem to have received attention) the effect of which is to check uprushes of moist air.

I have often noticed the cumulus clouds over a distant thunderstorm suddenly cease to rise and then disappear. Whilst rising, their outlines are clear and sharp. As soon as the rise ceases their outlines become indistinct. Then a partially transparent veil appears where the cumulus cloud was, and this fades away entirely, leaving the atmosphere clear. Why do cumulus clouds fail to rise so high as one would expect them to?

Warm saturated air near the earth's surface (warmed by proximity to the ground) rises and steadily cools. After the dew point is reached important results follow. Condensation takes place and the air is then prevented from falling in temperature so much as it would do if it were dry, and this would accelerate the velocity of the up current if it were not for the presence of the newly formed cloud particles. The small drops forming the cloud are floating in the air and contributing their quota to its weight. Condensation thus tends to check the ascent of the mass. When the particles become large enough to descend quickly, as rain or hail, they draw the air down with them and the uprush ceases.

The mechanical effect of falling rain, and the great density of a cloud due to the weight of the water particles in it, do not seem to have been generally considered.

Tintagil, Kew Gardens Road, Kew, Surrey, December 24

\section{Molecular Dimensions of Gelluloid.}

THE results of the experiments carried out by the Bureau of Standards, Washington (NATURE, December $x_{3}$, p. 86I), are of very great interest, but would be still more valuable if the exact composition of the "celluloid" were known. There is often confusion in referring to such words as " celluloid," " celloidin," " collodion" and the like. Celluloid, the basis of photographic film, is certainly a manufactured mixture of variable composition, containing among other ingredients a considerable percentage of camphor, the main ingredient being, of course, a soluble cellulose trinitrate, though cellulose acetate is now being increasingly used on account of its noninflammability. It is, therefore, scarcely correct to speak of the " molecular complex of celluloid" (unless " complex" is intended to cover a mixture of two or more compounds). "Celloidin" is the trade name of a carefully purified and soluble cellulose nitrate, probably approaching to a single chemical substance. Collodion is, of course, a solution of nitrocellulose in acetone, ether-alcohol, or some other organic solvent.

3 Lea Road,

Heaton Moor, near Stockport,

December I4.

ERratum.-In NATURE of October 4, p. 499, a letter from Prof. Was. Shoulejkin on " A New Method of Investigating Sea Waves" is subscribed "Technical High School, Moscow." The work described was carried out at the Physical Institution of the Science Institution of Moscow. 